\title{
Laboratory diagnosis of Mycoplasma pneumoniae infection
}

\section{Comparison of methods for the direct detection of specific antigen or nucleic acid sequences in respiratory exudates}

\author{
R. HARRIS ${ }^{1}$, B. P. MARMION ${ }^{2,3}$, G. VARKANIS ${ }^{3}$, T. KOK ${ }^{3}$, B. LUNN ${ }^{1}$ \\ AND J. MARTIN ${ }^{4}$ \\ ${ }^{1}$ School of Pharmacy and Medical Laboratory Science, S.A. Institute of \\ Technology, Adelaide ${ }^{2}$ Department of Pathology, University of Adelaide ${ }^{3}$ Division \\ of Medical Virology, Institute of Medical and Veterinary Science, Adelaide \\ ${ }^{4}$ Department of Pulmonary Medicine, Adelaide Children's Hospital
}

(Accepted 19 July 1988)

\section{SUMMARY}

The efficiency of the direct detection of Mycoplasma pneumoniae in respiratory exudates by an antigen capture, indirect enzyme immunoassay (Ag-EIA), has been compared with its detection with a cDNA probe ('Gen-Probe assay') directed against the specific ribosomal RNA sequences of the organism ('Mycoplasma pneumoniae Rapid Diagnostic System', Gen-Probe, San Diego, California).

Both assays showed excellent specificity against a range of mycoplasma species suspended in negative nasopharyngeal aspirates; only $M$. pneumoniae and $M$. genitalium reacted. In experiments with graded doses of viable $M$. pneumoniae cells suspended in negative nasopharyngeal aspirate, the Gen-Probe assay was more sensitive than Ag-EIA; detection limits were respectively $2 \times 10^{3}$ c.f.u. $/ \mathrm{ml}$ $\left(3.2 \times 10^{5}\right.$ genomes $)$ and $2.5 \times 10^{4}$ c.f.u. $/ \mathrm{ml}\left(4 \times 10^{6}\right.$ genomes $)$; detection levels 10-100 times less sensitive than culture.

The two assays were also tested on nasopharyngeal aspirates or sputum specimens from 90 patients with respiratory infection; 67 of these were culture- or seronegative for $M$. pneumoniae and 23 were culture- or seropositive. Ag-EIA detected $21(91 \%)$ of the latter but the Gen-Probe assay detected only $5(22 \%)$. Both assays were negative with the 67 culture-/sero-negatives ; there were no GenProbe assay positive/Ag-EIA negatives.

Overall, it is concluded that although Ag-EIA and the Gen-Probe assay are effective substitutes for culture as a diagnostic procedure, there is a significant problem with samples which are culture-negative and from patients who have good serological evidence of current infection. Possible reasons for the disparity between the two assays are advanced.

Please address correspondence and reprint requests to Professor B. P. Marmion, Medical Virology, Institute of Medical and Veterinary Science, Box 14, Rundle Mall Post Office, Adelaide, South Australia, 5000. 


\section{INTRODUCTION}

In a previous paper (Kok et al. 1988) we have outlined the clinical importance of Mycoplasma pneumoniae infection and the shortcomings of existing methods for its laboratory diagnosis. It also described the development and validation of an indirect enzyme immunoassay (Ag-EIA) for the detection of $M$. pneumoniae antigen in samples of nasopharyngeal mucus or sputum from suspected cases of infection.

A possible alternative or supplementary approach to direct diagnosis of infection by antigen detection is the assay of the specific nucleotide sequences of the mycoplasma in the respiratory exudates. The sequences assayed could either be genomic, or those ribosomal sequences specific for the organism. The first may be detected with probes prepared from genome fragments cloned in a plasmid or other vector; $M$. pneumoniae DNA would be detected by 'dot blot' hybridization with ${ }^{32} \mathrm{P}$-labelled DNA from these inserts as probes, followed by autoradiography and counting of the radioactive emissions from the 'dot blots' (Hyman, Yogev \& Razin, 1987). Specific ribosomal sequences may be assayed with the 'Mycoplasma pneumoniae Rapid Diagnostic System' (Gen-Probe, San Diego, California), which is based on the detection of $M$. pneumoniae ribosomal RNA (rRNA) by in-solution hybridization to a ${ }^{125} \mathrm{I}-\mathrm{cDNA}$ probe.

This report compares the specificity and sensitivity of detection by Ag-EIA and by the M. pneumoniae Rapid Diagnostic System relative to detection of the organism by culture or the demonstration of infection by a serological response in the patient.

\section{MATERIALS AND METHODS}

Laboratory confirmation of $\mathrm{M}$. pneumoniae infection

Methods for the detection of complement fixing (CF) and specific IgM antibody to $M$. pneumoniae, for the Ag-EIA and for culture of the organism were those described by Kok et al. (1988).

Gen-Probe M. pneumonia rapid diagnostic sytem. Specimens of nasopharyngeal mucus (NPA) or sputa were treated to reduce viscosity, and the organisms from $1.0 \mathrm{ml}$ were concentrated by centrifugation. A lysing solution from the kit was added to release ribosomal RNA. The rRNA was then hybridized in solution to a specific ${ }^{125} \mathrm{I}$-cDNA probe for $1 \mathrm{~h}$ at $72^{\circ} \mathrm{C}$. The hybrid formed was adsorbed to hydroxyl-apatite and collected by centrifugation for estimation of radioactivity in a gamma counter. A small number of tests (e.g. 1-5) can be completed in less than $2 \mathrm{~h}$.

The activity was expressed in terms of the ratio (specimen ratio) between radioactivity found following hybridization of the probe and the test specimen as compared with that found following hybridization with the negative control supplied with the kit, namely,

$$
\text { specimen ratio }=\frac{\text { specimen c.p.m. - background c.p.m. }}{\text { negative control c.p.m. - background c.p.m. }} .
$$

The recommended 'cut-off' point is a ratio of $\geqslant 3 \cdot 0$, and indicates that the test sample is positive for $M$. pneumoniae. 
Although the kit is described by the manufacturer for use with material eluted from throat swabs, it was found that mycoplasmas in nasopharyngeal aspirates (NPA) (in both simulated specimens and those from natural infections) were readily detected (see Fig. 1 and Tables 1-3).

Growth and preparation of M. pneumoniae and other mycoplasma strains and quantitation

This followed the methods described in Kok et al. (1988).

\section{Collection, preparation and examination of clinical and simulated specimens}

Clinical specimens. Details of the patients, methods of sampling, and details of viral diagnosis and culture of $M$. pneumoniae are given in Kok et al. (1988). The majority of nasopharyngeal aspirates (NPA) examined were collected from children aged 1-15 years at the Adelaide Children's Hospital. Other NPA and sputa were from the sources indicated in Kok et al. (1988). In all, 90 specimens were collected and tested.

Artificially infected specimens ('simulated positives'). A pool of nasopharyngeal aspirates, previously shown to be culture- and antigen-negative for $M$. pneumoniae, was prepared. For the nucleic acid detection assays a culture of $\boldsymbol{M}$. pneumoniae organisms was diluted in the pool to give final concentrations, ranging from $10^{1}$ to $10^{6}$ c.f.u. $/ \mathrm{ml}$, in half-log intervals. Mycoplasma-free HeLa 229 cells at a final concentration of $10^{5}$ cells per $\mathrm{ml}$ were also added to the specimens depicted in Fig. 1. Aliquots $(0.1$ or $1.0 \mathrm{ml})$ of these simulated positive specimens were used in the Gen-Probe system. For the antigen capture assay, similar preparations of $M$. pneumoniae were made, except for that the simulated positive samples were diluted with an equal volume of $5 \%$ skim milk-phosphate-buffered saline containing $0.5 \%$ Tween 20 prior to Ag-EIA assay (Kok et al. 1988).

\section{Determination of DNA content of cells}

DNA determination on whole mycoplasma cells and purified DNA utilized the simple and very sensitive DAPI-mediated fluorescence methods of Legros \& Kepes (1985) and Kapuscinski \& Skoczylas (1977). Additionally, because of interference by at least one SP-4 medium component (phenol red), organisms were pelleted at $10000 \mathrm{~g}$ for $20 \mathrm{~min}$ at $4{ }^{\circ} \mathrm{C}$, resuspended in PBS, pelleted and finally resuspended in PBS before the DNA determinations. Salmon sperm DNA was used as a standard. The specificity of the fluorescence as an indicator of DNA concentration was checked by treating the $M$. pneumoniae cells with toluene to open the membranes and incubating the treated preparations with DNAase 1 ; this showed that $>\mathbf{7 0} \%$ of the fluorescent signal was abolished.

The concentration of purified salmon sperm DNA was determined in a spectrophotometer calibrated so that one absorbance unit at A260 was equivalent to $50 \mu \mathrm{g} / \mathrm{ml}$ of DNA.

Determination of the number of M. pneumoniae genomes/c.f.u.

The DAPI method was used to determine the relation between one colonyforming unit (c.f.u.) of $M$. pneumoniae (assayed as described by Kok et al. 1988) and the number of genomes; a figure of 160 genome copies per c.f.u. was obtained 
(average of three determinations), taking the genome size as $7 \cdot 3 \times 10^{5}$ base pairs or $4.8 \times 10^{8}$ daltons, Bak et al. (1969). The ratio of genomes per c.f.u. is (perhaps) suprisingly high, but $M$. pneumoniae tends to grow in fluid media as small colonies and multigenome cells rather than as dispersed suspensions of single cells each with a single genome. It would be necessary to filter the suspensions to obtain a preparation of single cells (Lemcke, 1971). It is probable that titrations by Collier \& Clyde (1974) of the c.f.u. in sputa of naturally infected patients would similarly underestimate the number of genomes present.

\section{RESULTS}

\section{Specificity of Ag-EIA and Gen-Probe hybridization assays}

A comparison of the reaction profiles obtained with the Ag-EIA and the GenProbe assays and a range of human and animal mycoplasma species is set out in Table 1. Most mycoplasmas were tested at concentrations of $\geqslant 10^{6} \mathrm{c.f} . \mathrm{u} . / \mathrm{ml}$. The pattern of reactions with the Mycoplasma spp. was consistent between the two assays; both reacted most strongly with $M$. pneumoniae and to a lesser extent with M. genitalium. Ag-EIA gave the most decisive differentiation between these two species. All other reactions with mycoplasmas were below the 'cut-off' values nominated for both of the assays.

Comparison of sensitivity of M. pneumoniae detection by Ag-EIA and by the GenProbe assay for specific ribosomal RNA sequences

A range of dilutions of a culture of $\mathrm{FH}$-Liu strain of $M$. pneumoniae was prepared in a pool of nasopharyngeal aspirates. This gave final concentrations from $1 \times 10^{6}$ c.f.u. $/ \mathrm{ml}$ to $1 \times 10^{1}$ c.f.u. $/ \mathrm{ml}$; a larger number of replicates, prepared from more closely spaced dilutions of the mycoplasma, were tested around the (probable) end-point for the Ag-EIA, namely $2.5 \times 10^{4} \mathrm{c.f} . \mathrm{u} / \mathrm{ml}$. Each dilution was cultured by inoculation of $0.1 \mathrm{ml}$ into the HeLa 229 monolayer culture/agar system, also by Ag-EIA $(0.1 \mathrm{ml})$ and by the Gen-Probe hybridization $(1.0 \mathrm{ml})$ assay. Results are shown in Table 2 . As might be anticipated, culture was the most sensitive; positive cultures were obtained on sub-inoculation from the $1 \times 10^{2}$ c.f.u./ml dilutions. It appears that 10-100 c.f.u. are required to initiate growth in the cell monolayer/agar culture system. From the DAPI determinations of $\boldsymbol{M}$. pneumoniae DNA described in Methods, it was calculated that 1 c.f.u. is equivalent to 160 genomes. So assuming one genome per organism, some 1600 organisms are required to initiate growth in culture. This poor plating efficiency might be due either to the use of culture media that are still suboptimal-although the Liu strain was well adapted to growth in the culture system - or to the presence of cell aggregates, multigenome cells or non-viable organisms in the seed cultures. The Gen-Probe assay, used with the reaction volume of $1.0 \mathrm{ml}$ specified by the manufacturer, detected organisms at $1 \times 10^{3}$ c.f.u/ml (or $2 \times 10^{5}$ genomes) and Ag-EIA, using a $0.1 \mathrm{ml}$ sample volume, at $2.5 \times 10^{4}$ c.f.u. $/ \mathrm{ml}$ (or $4 \times 10^{6}$ genomes).

Fig. 1 gives titration curves for the Ag-EIA and Gen-Probe assays using the $\mathrm{Ca}$ strain of $M$. pneumoniae. The superior sensitivity of the Gen-Probe system was reduced relative to $\mathrm{Ag}$-EIA when equivalent sample volumes of $0.1 \mathrm{ml}$ were used 
Table 1. Specificity of antigen-capture enzyme immunoassay and Gen-Probe hybridization assay when tested with a range of mycoplasma species of human and animal origin.

(Testing was in a volume of $1.0 \mathrm{ml}$ for the Gen-Probe kit and $0.1 \mathrm{ml}$ for Ag-EIA and at a final concentration of $>10^{6}$ c.f.u. $/ \mathrm{ml}$, diluted in a pool of negative nasopharyngeal aspirates.)

$\begin{array}{lcc} & \begin{array}{c}\text { Antigen- } \\ \text { capture } \\ \text { EIA, } \\ \text { percentage } \\ \text { specific }\end{array} & \begin{array}{c}\text { Gen-Probe } \\ \text { ribosomal } \\ \text { RNA assay } \\ \text { specimen }\end{array} \\ \text { binding } & \text { ratios } \\ \text { Mycoplasma species } & 68 & 40 \cdot 7 \\ M \text {. pneumoniae (Liu strain) } & 1 & 1 \cdot 7 \\ M \text {. orale } & 0 & 1 \cdot 3 \\ M . \text { buccale } & 1 & 1 \cdot 4 \\ M \text {. faucium } & 2 & 1 \cdot 6 \\ M . \text { salivarium } & 2 & 1 \cdot 5 \\ M \text {. fermentans } & 9 & 23 \cdot 6 \\ M . \text { genitalium } & 0 & 1 \cdot 7 \\ M \text {. hominis } & 0 & 1 \cdot 2 \\ M \text {. hyorhinis } & 0 & 1 \cdot 3 \\ \text { A. laidlawii } A & 1 & 1 \cdot 7 \\ \text { A. laidlawii } B & <2 & 0 \cdot 9 \\ \text { E. coli K12 suspension } & <2 & 1 \cdot 1 \\ \text { Streptococcus MG suspension } & <2 & 0 \cdot 8 \\ \text { HeLa 229, disrupted cells } & <2 & 1 \cdot 5 \\ \text { Pool of negative nasopharyngeal } & & \\ \text { aspirates } & 5 & \geqslant 3 \\ \text { Cut-off value } & & \end{array}$

in both tests - end-points were at $2.7 \times 10^{4}$ c.f.u. $/ \mathrm{ml}$ for Ag-EIA and $9.8 \times 10^{3}$ c.f.u./ml for the Gen-Probe assay.

Attempts to concentrate the organism from $1.0 \mathrm{ml}$ samples for antigen detection led to a 2- to 3 -fold increase in sensitivity, but the centrifuged pellets required much sonication and detergent treatment before they were acceptable for use in the test. These additional steps were an unacceptable complication in a routine diagnostic procedure.

Comparative sensitivity of detection of M. pneumoniae by Ag-EIA and Gen-Probe hybridization assay with clinical specimens

Nasopharyngeal aspirates or sputum samples from 90 patients with a respiratory tract infection during the 1986-7 prevalence of $M$. pneumoniae infection were tested by Ag-EIA and by the $M$. pneumoniae rapid diagnostic system (Gen-Probe) (Table 3). Twenty-three patients had either culture or serological evidence of current infection with $M$. pneumoniae, or both. The remaining 67 patients were culture-negative and had no serological evidence of current infection (details in Table 3 : the total group of patients has been divided into three subgroups A, B, C in order of decreasing weight of positivity with the standard diagnostic markers of culture and serology).

In subgroup A of Table 3, there were two culture-positive patients, and each had clear serological evidence of infection. Both were positive by Ag-EIA, but 
Table 2. Comparison of the sensitivity of M. pneumoniae culture, Ag-EIA and GenProbe hybridization assays with specimens artificially infected with $\mathbf{M}$. pneumoniae

(Assay results are shown as the numbers of specimens giving positive results relative to the total specimens at each concentration of organism.)

\begin{tabular}{|c|c|c|c|}
\hline $\begin{array}{l}\text { M. pneumoniae } \\
\text { concentration } \\
\text { (c.f.u./ml } \\
\text { in specimen) }\end{array}$ & $\begin{array}{c}\text { Culture } \\
\text { (No. + ve/total) }\end{array}$ & $\begin{array}{c}\text { Antigen-capture } \\
\text { Ag-EIA } \\
\text { (No. + ve/total) }\end{array}$ & $\begin{array}{c}\text { Gen-Probe } \\
\text { rRNA assay } \\
\text { (No. + ve/total) }\end{array}$ \\
\hline $1 \times 10^{6 *}$ & $2 / 2$ & $2 / 2$ & $2 / 2$ \\
\hline $1 \times 10^{5}$ & $2 / 2$ & $2 / 2$ & $2 / 2$ \\
\hline $5 \times 10^{4}$ & $10 / 10$ & $10 / 10$ & $10 / 10$ \\
\hline $2.5 \times 10^{4}$ & $10 / 10$ & $10 / 10$ & $10 / 10$ \\
\hline $1 \times 10^{4}$ & $10 / 10$ & $0 / 10$ & $10 / 10$ \\
\hline $7 \cdot 5 \times 10^{3}$ & $5 / 5$ & $0 / 5$ & $5 / 5$ \\
\hline $5 \times 10^{3}$ & $3 / 3$ & $0 / 3$ & $3 / 3$ \\
\hline $1 \times 10^{3}$ & $2 / 2$ & $0 / 2$ & $2 / 2$ \\
\hline $1 \times 10^{2}$ & $2 / 2$ & $0 / 2$ & $0 / 2$ \\
\hline $1 \times 10^{1}$ & $0 / 2$ & $0 / 2$ & $0 / 2$ \\
\hline Medium control & $0 / 2$ & $0 / 2$ & $0 / 2$ \\
\hline $\begin{array}{l}\text { Assay volume } \\
(\mathrm{ml}) \dagger\end{array}$ & $0 \cdot 1$ & $0 \cdot 1$ & $1 \cdot 0$ \\
\hline
\end{tabular}

* Final concentration (c.f.u./ml) in the pool of negative nasopharyngeal aspirates.

$\dagger$ Actual volume used in each assay.

only one in the Gen-Probe assay. In subgroup B, all of whom had clear serological evidence of current infection, there were 19 antigen-positives and four were GenProbe assay-positive.

\section{DISCUSSION}

The introduction and the paper by Kok et al. (1988) summarized the inadequacies of current laboratory methods for detection of $M$. pneumoniae infection and the need for direct detection methods for antigen or specific nucleotide sequences in respiratory exudates. An antigen-capture EIA (Ag-EIA) has been developed (Kok et al. 1988), and in the present report has been compared with a recently developed hybridization assay for a specific rRNA sequence(s) ('Mycoplasma pneumoniae Rapid Diagnostic System'; Gen-Probe).

Both Ag-EIA and the Gen-Probe rRNA assay proved to be highly specific reacting only with $M$. pneumoniae and $M$. genitalium. Ag-EIA gave the clearest differentiation between the two species. There was no reaction with nine other species of mycoplasmas from human or animal sources. The antigenic crossreactivity and nucleic acid sequence homology between $M$. pneumoniae and M. genitalium is well recognised (Kenny \& Cartwright, 1984; Lind et al. 1984; Clyde \& $\mathrm{Hu}, 1986$ ).

In initial experiments with simulated positive samples -- i.e. pools of negative nasopharyngeal aspirates seeded with known numbers (c.f.u.) of laboratory strains of M. pneumoniae and HeLa 229 cells - the Gen-Probe rRNA assay proved to be more sensitive than Ag-EIA and approached culture of the organism in sensitivity (Figure 1 and Table 2). 


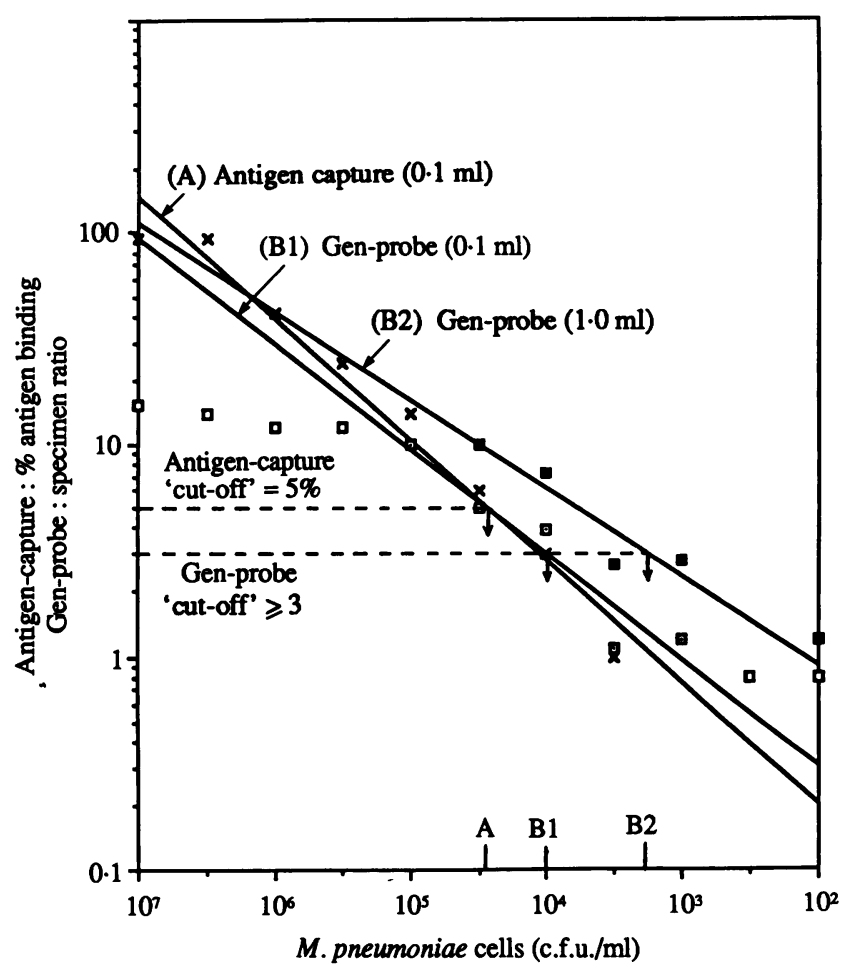

Fig. 1. End-points of detection at various concentrations (c.f.u./ml) of the Ca strain of M. pneumoniae in a pool of negative nasopharyngeal aspirates with $10^{4} \mathrm{HeLa} 229$ cells/ $\mathrm{ml}$ by Ag-EIA and Gen-Probe hybridization. Lines of best fit were calculated by the method of least squares. Each of the Gen-Probe assay lines of best fit utilized data from the non-plateau regions of the titration curves.

End-point sensitivities of $M$. pneumoniae cell detection are: (A) Ag-EIA (0.1 ml), $2.7 \times 10^{4}$ c.f.u. $/ \mathrm{ml}$; (B1) Gen-Probe $(0.1 \mathrm{ml}), 9.8 \times 10^{3}$ c.f.u. $/ \mathrm{ml} ;(B 2)$ Gen-Probe $(1.0$ $\mathrm{ml}), 1.8 \times 10^{3}$ c.f.u. $/ \mathrm{ml}$. Line equations: (A) $Y=0.0144 \times 10^{0.5737 x}(R=0.99)$; (B1) $Y=$ $0.0308 \times 10^{0.4981 X}(R=0.96)$; (B2) $Y=0.1311 \times 10^{0.4181 X}(R=0.93)$.

From our experiments with simulated positive samples and a small number of culture-positive specimens from natural infections, we readily accept the claims in the Gen-Probe product leaflet that there is a clear and close agreement between a positive culture for $M$. pneumoniae and a positive Gen-Probe rRNA assay, whether with simulated positive samples or with eluates from throat swabs taken from $M$. pneumoniae infected patients (Shaw \& Weiner, 1987; Dular, Kajioka \& Kasatiya, 1988). Our series of clinical specimens included only two which were positive in culture. Both of these were positive by Ag-EIA and one by Gen-Probe rRNA assay.

However, tests with the Gen-Probe assay on a selected series of samples from 21 patients who were culture-negative but had compelling serological evidence of current infection, revealed a different and somewhat unexpected pattern. Nineteen $(90 \%)$ were positive by Ag-EIA but only $4(19 \%)$ by Gen-Probe rRNA assay. It should be noted that the proportion of Ag-EIA-positive samples to the total of those from culture-negative, seropositive patients is higher than that reported for such patients by Kok et al. (1988); antigen-positive samples were to some extent 
Table 3. Relation between the results of culture and serodiagnostic tests for M. pneumoniae infection and those of antigen capture $(A g-E I A)$ and the Gen-Probe assay for specific ribosomal RNA sequences of the mycoplasma

rRNA

hybridization

Ag-EIA

Subgroup Diagnostic markers

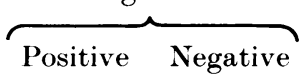

\begin{tabular}{|c|c|}
\hline A & $\begin{array}{l}\text { Culture-positive* } \\
\text { Seropositive }{ }^{\dagger}\end{array}$ \\
\hline$B$ & $\begin{array}{l}\text { Culture-negative* } \\
\text { Seropositive } †\end{array}$ \\
\hline $\mathrm{C}$ & $\begin{array}{l}\text { Culture-* and } \\
\text { seronegative }\end{array}$ \\
\hline
\end{tabular}

2

0

Positive Negative Total

Total

19

0

9

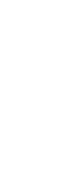

2

67
(Gen-Probe)

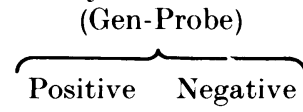

1

4

0
1

67
2

90

* Culture of M. pneumoniae on cell monolayer/agar subculture.

+ Fourfold or greater increase in CF or specific IgM antibody or a specific IgM antibody titre of $\geqslant 320$. No evidence of current infection with respiratory viruses.

$\ddagger$ CF antibody $<10 ;$ specific IgM antibody $\leqslant 80$.

selected for comparison with the Gen-Probe assay. A comparison of Ag-EIA positive and negative proportions with those of the Gen-Probe assay, for the total group of 90 samples, gives $\left.\chi^{2}=11.5, P<0.0005\right)$. The disparity between Ag-EIA and Gen-Probe assays requires some explanation, as the evidence presented by Kok et al. (1988) is considered to establish the specificity of the Ag-EIA for $M$. pneumoniae infection, and the criteria set for serological evidence of current infection are stringent.

We consider that at least three explanations are possible. The first is that the ribosomal RNA sequences constituting the target for Gen-Probe's cDNA probe had decayed on storage of the specimens; the high cost of the kits, the limited shelf life of the $\mathrm{I}^{125}$ isotope and the transit time for delivery of the kits from the United States to Australia sometimes made it necessary to store the clinical samples for batch testing. The possibility of degradation of the RNA target on storage has been investigated by multiple freezing and thawing of specimens artificially seeded with $M$. pneumoniae and also by exposing them to RNAase during the freezing and thawing process and, finally, by distrupting the organisms with toluene before treating them with ribonuclease. Freeze-thaw cycles did not significantly reduce target reaction (specimen ratios were frequently unchanged and when altered did not exceed $8 \%$ per freeze-thaw cycle). Short exposures (1 $\mathrm{h}$ at $37^{\circ} \mathrm{C}$ ) of the intact organism to RNAase did not reduce the target. Prolonged exposure to RNAase, especially when the specimen was pretreated with small amounts of toluene to disrupt the cell membrane, markedly reduced target activity. Thus, target instability on ordinary storage with freezing and thawing does not appear to be a problem.

A second possible explanation is that the Gen-Probe-negative, Ag-EIApositive cases represent infections with a mycoplasma or other organism sharing antigens with $M$. pneumoniae, but with no ribosomal sequence homology. This seems highly unlikely, as the only mycoplasma which cross-reacts antigenically 
with $M$. pneumoniae is $M$. genitalium, and this, in our tests, also shows extensive cross-hybridization, both by Gen-Probe and by DNA 'dot blot' hybridization (Hyman, Yogev \& Razin, 1987 and our own unpublished data). Moreover, the data presented by Kok et al. (1988) also make it highly unlikely that a hypothetical, antigenically cross-reacting bacterium or other prokaryote could explain both the Ag-EIA results and the patients' serological reactions with standard M. pneumoniae antigens in a group of cases with a disease characteristic of $M$. pneumoniae infection.

A third possible explanation, which we presently favour, is that the antigen and rRNA sequences are broken down at different rates in the respiratory tract of the patients as they recover from infection. Malaise and debility from M. pneumoniae infection sometimes develop slowly, and patients may not present for sampling until they reach mid-disease, at which time the cellular and immune responses in the respiratory tract may be active and a course of broad-spectrum antibiotics may have been given. The protein and glycolipid antigens of the organism may have a better survival potential in the respiratory tract than the rRNA, once the organisms are damaged by immune reactions or antibiotics and membrane integrity is lost with access of nucleases to the rRNA target. Indeed, the close concordance between positive culture ( = viable, membrane-intact organism) and Gen-Probe assay positivity, and on the other hand, the divergence between the Gen-Probe assay and the Ag-EIA in the culture-negative/seropositive group of patients is in agreement with this view. It may be that genomic DNA would be more stable under conditions of natural infection, thus the value of probes to genomic sequences of $M$. pneumoniae merits investigation with clinical specimens.

In general, the findings again raise the question, discussed by Kok et al. (1988), of the 'gold standard ' against which the efficiency of a direct diagnostic test should be compared. We submit that this should not be limited to comparisons with the culture of the organism but should also take account of the culture-negative patient with good serological evidence of a current infection.

We acknowledge (1) support provided under the National Biotechnology Research Program which is administered by the Commonwealth Department of Industry, Technology and Commerce; the Technology Innovation Fund, Department of State Development, South Australia; the Channel 7 Children's Research Foundation of South Australia; and the generous support of Mrs Isabel Millner; (2) help from Sister Jill Thompson for specimen collection at the Adelaide Children's Hospital; (3) secretarial help from Mrs Julie Maylin.

Dedication. This work is dedicated to Noel Millner, in memoriam.

\section{REFERENCES}

Bak, A., Black, F., Christiansen, C. \& Freundt, E. (1969). Genome size of mycoplasmal DNA. Nature 224, 1209-1210.

ClYDE, W. JR \& Hu, P-C. (1986). Antigenic determinants of the attachment protein of Mycoplasma pneumoniae shared by other pathogenic mycoplasma species. Infection and Immunity 51, 690-692. 
Collier, A. \& Clyde, W. JR (1974). Appearance of Mycoplasma pneumoniae in lungs of experimentally infected hamsters and sputum from patients with natural disease. American Review of Respiratory Disease 110, 765-773.

Dular, R., Kajioka, R. \& Kasatiya, S. (1988). Comparison of Gen-Probe commercial kit and culture technique for the diagnosis of Mycoplasma pneumoniae infection. Journal of Clinical Microbiology 26, 1068-1069.

Hyman, H., Yogev, D. \& Razin, S. (1987). DNA probes for detection and identification of Mycoplasma pneumoniae and Mycoplasma genitalium. Journal of Clinical Microbiology 25, 726-728.

Kenny, G. \& Cartwright, F. (1984). Immunoblotting for determination of the antigenic specificities of antibodies to the Mycoplasmatales. Israel Journal of Medical Sciences 20, 908-911.

Kok, T-W., Varkanis, G., Marmion, B. P., Martin, J. \& Esterman, A. (1988). Laboratory diagnosis of Mycoplasma pneumoniae infection. 1. Direct detection of antigen in respiratory exudates by enzyme immunoassay. Epidemiology and Infection 101, 669-684.

Legros, M. \& KePES, A. (1985). One step fluorometric microassay of DNA in prokaryotes. Analytical Biochemistry 147, 497-502.

LEMCKE, R. (1971). Sizing small organisms. Nature 229, 492-493.

Lind, K., Lindhardt, B., Schutten, H., Blom, J., Christiansen, C. (1984). Serological crossreactions between Mycoplasma genitalium and Mycoplasma pneumoniae. Journal of Clinical Microbiology 20, 1036-1043.

Shaw, S. B. \& Weiner, L. B. (1987). Clinical studies summary report. The Gen-Probe rapid diagnostic system for Mycoplasma pneumoniae (personal communication). 